Article

\title{
Knee-Assistive Robotic Exoskeleton (KARE-1) Using a Conditionally Singular Mechanism for Industrial Field Applications
}

\author{
Hyung Joo Kim ${ }^{1}{ }^{(\mathbb{C}}$, Jaeho $\mathrm{Noh}^{2}$ and Woosung Yang ${ }^{2, * \mathbb{C}}$ \\ 1 Hyundai Motor Company, Hwaseong 18280, Korea; kimhjoo@gmail.com \\ 2 School of Robotics, Kwangwoon University, Seoul 01897, Korea; causeim@gmail.com \\ * Correspondence: dreamrize@kw.ac.kr; Tel.: +82-2-940-8115
}

Received: 9 June 2020; Accepted: 16 July 2020; Published: 27 July 2020

check for updates

\begin{abstract}
With the aging demographic of today's society, the need for robotic exoskeletons is expected to increase as they can compensate for declining physical strength in the physically impaired. In this study, an assistive robotic exoskeleton for the knee joint with fairly low energy consumption is proposed for industrial applications. The knee-assistive robotic exoskeleton (KARE-1) was designed to support a human body during production line tasks. The KARE-1 is based on a four-bar link mechanism with a rotary actuator and gas spring to accommodate a high power-to-weight ratio. By taking advantage of the utilized singular configuration of the four-bar linkage, this novel design is able to efficiently support the weight of the human body. The selected singular configuration allows this device to support the knee joint in the load-bearing stages of static sitting as well as during the motion between standing and sitting. The proposed device is further able to move freely along with the knee during walking movements. The proposed design was verified through a series of numerical simulations and through human subject testing at an industrial workplace.
\end{abstract}

Keywords: exoskeleton; wearable robot; assist robot; singular mechanism

\section{Introduction}

Interest in the use of robotic devices for assisting both healthy and disabled people with various activities has been growing over the past two decades. Robotic exoskeletons have been developed to augment or assist the physical functions of humans in industry as well as daily life. These are often controlled by advanced technologies developed specifically for human-robot interaction (HRI). Physical HRI is capable of decoding and reacting to a human's motion-based intentions through physical contact with the wearer.

Advancements in robotic exoskeleton technologies were demonstrated by a number of wearable robots: BLEEX [1] showed success in augmenting human load-carrying performance in field testing for military applications, while iHAS [2], Noonee [3], Robo-mate project [4], and BobyWeight Assist devices [5] have done the same for industry applications, and the Hybrid Assist Limb [6] shows promise for rehabilitation. Following these achievements, a new modular type [3-5] of human-assistive device was developed to alleviate the physical workloads placed on workers.

The market for robotic exoskeletons is expected to increase with society's aging demographics. In industry, robotic exoskeletons may be used to assist physically impaired individuals working for prolonged periods, helping to improve their productivity, safety, and quality of work. These factors will reduce production costs as well as the risk of injury [6].

A number of new technologies in physical HRI have been introduced to address existing safety issues. Highly back-drivable mechanisms can accommodate safe interaction with human operators $[7,8]$. 
Implementing passive elements $[9,10]$ and pneumatic actuators [11,12] into device design enables improved mechanical compliance in a robot. The design and control of a variable stiffness actuator can also help to actualize fast and safe physical HRI $[13,14]$. Alternative approaches for modifying physical HRI systems have also been proposed, including a knee brace based on a magnetorheological actuator by Chen et al. [15], an electrorheological fluid actuator for a knee orthotic device [16], a quasi-passive knee exoskeleton using a cable-driven series elastic actuator by Kamaran et al. [17], a knee-assistive device driven by a series elastic actuator by Pratt et al. [18], and multiple variable stiffness actuators proposed by Nikos et al. [19].

These existing designs, however, have encountered difficulties controlling motion generation (corresponding to the wearer's intention) and power assistance (reducing stress on the knee). Moreover, these devices require a large power capacity, as the actuator is driven continuously while the wearer is walking or sitting. This causes energy consumption problems over the course of long-term operation.

In contrast to existing devices, this study proposes an alternative four-bar mechanism combining passive and active actuators so that this device can assist the wearer's body weight using a small torque input in sitting and can provide no resistance in walking. This work designs and proposes a knee-assistive robotic exoskeleton (KARE-1) for application to the industrial field, which can completely support a worker's body weight in various sitting postures.

To this end, it is worth noting that, differently from the previous work [20], three representative kinds of four-bar mechanism-based assistive devices were analyzed and compared to optimize torque generation and reduce energy consumption. Simulation results of the proposed system exhibited the requisite dynamic properties, including torque, displacement, and force amplification. Finally, the effectiveness of the proposed approach was verified experimentally using a real assistive robot. Industry applicability was further confirmed by measuring muscle activation during testing with a human subject.

\section{Design Requirements}

When working at sitting posture the leg muscle forces are exerted consistently against the gravity forces, and an assistive body support device that can relieve the knee muscle loadings during sitting would be of great help to the physical workers. Furthermore, for industrial application the body support device needs to be controlled adaptively to workers of various body sizes, and to working conditions of different sitting postures, and even to walking motion.

There are two specific examples of body weight support devices developed so far. One type is a locking mechanism that sustains the body weight at an arbitrary seated position [3]. A worker could get the benefits of saving muscle effort in static situations using the locking mechanism by making use of passive mechanisms. However, this has some drawbacks in dynamic situations. For example, when a worker wants to change a body posture, the supporting mechanism can either provide some assistive torque to the human body, or make itself back-drivable so that a worker can change its configuration very easily as wished. The other type is a support device that assists a wearer's body continuously using actuators, for example the body weight support device developed by Honda [5]. The objective of this device was to reduce the knee muscle loadings of the worker in an arbitrary posture or motion by a preselected assistance ratio. However, due to its high energy consumption, it had to provide a limited amount of assistant torques to the knee to keep a long operation time.

This study proposed a body support device that featured the advantages of both types, i.e., to support a human body with high energy efficiency during sitting, and to make its joints back-drivable so that a worker could change its configuration very easily as wished. As more wearable assistive devices such as the similar examples [3-5] illustrated in the introduction are developed for production line or nonuniform assembly line work, unburdening carrying and keeping heavy parts from physical workers, the need for this self-supporting device would increase, thereby relieving muscle pain and fatigue due to keeping awkward postures or routing repeatedly. In order to secure 
the applicability of a proposed device in industrial workers, a target scenario was set as in Figure 1 and Table 1. The performance goals of the robotic knee-assistive device are set as follows:

1. Minimize the number of active actuators.

2. Assist up to a maximum body weight of $85 \mathrm{~kg}$.

3. Energy consumption less than $1 \mathrm{Wh}$.

4. Specific operating conditions:

- Moving distance $<15 \mathrm{~m}$

- Walking speed $<4 \mathrm{~km} / \mathrm{h}$

- Working time in sitting pose $>$ in walking motion

- $\quad$ Phase transition between sitting and walking $>1$ cycle/min

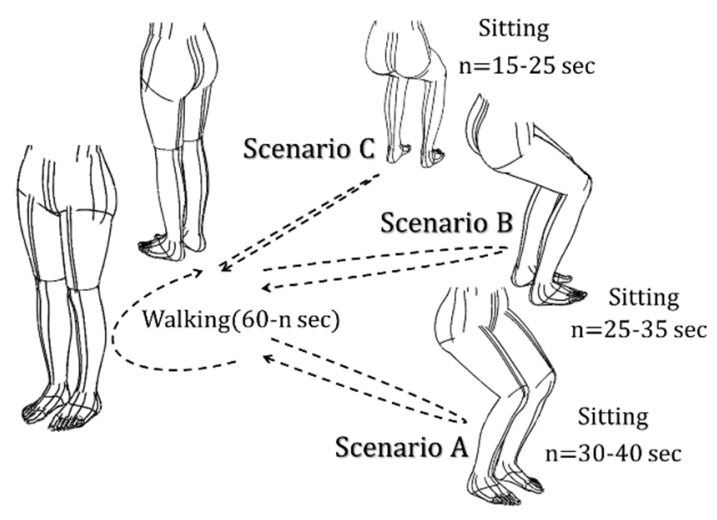

Figure 1. Working scenario.

Table 1. Knee Joint Working Conditions and Performance Requirements.

\begin{tabular}{cccc}
\hline & - & Min. & Max. \\
\hline \multirow{2}{*}{ Objective } & Walking Speed & - & $4 \mathrm{~km} / \mathrm{h}$ \\
Working Scenario & Walking Distance & $10 \mathrm{~m}$ & $15 \mathrm{~m}$ \\
& Sitting Time(n) & $30 \mathrm{~s}$ & $40 \mathrm{~s}$ \\
\hline \multirow{3}{*}{ Performance } & Range of Motion & $0 \mathrm{deg}$ & $90 \mathrm{deg}$ \\
Requirements & Peak Torque [21,22] (Normalized) & $0.44 \mathrm{Nm} / \mathrm{kg}$ & $0.77 \mathrm{Nm} / \mathrm{kg}$ \\
& Peak Torque (85 kg of person) & $38 \mathrm{Nm}$ & $65 \mathrm{Nm}$ \\
& Peak Speed & - & $400 \mathrm{deg} / \mathrm{s}$ \\
\hline
\end{tabular}

The specific conditions of the work scenario referred to the data in the literature, on physical workers at an assembly line and workshop. Their weights were between $72 \mathrm{~kg}$ to $101 \mathrm{~kg}$, and heights were between $173.6 \mathrm{~cm}$ and $183.3 \mathrm{~cm}$ (the 50th to 95th percentile male) [21]. In the paper the working conditions of workplaces were categorized into three scenarios (A, B, and C in Figure 1) according to the tasks. Among these three scenarios, $\mathrm{A}$ is considered the most arduous, since it features the longest sitting time. In this study the target condition for the body support device was set to be scenario A. More specifically, the range of knee motion during sitting was set to be between 0 to 90 degrees, and the walking speed was set to be $4 \mathrm{~km} / \mathrm{h}$.

Prior studies [22,23] reported that the required knee torque was between 0.44 and $0.77 \mathrm{Nm} / \mathrm{kg}$ during the transition motion between sitting and standing. Dynamic loadings of the knee joint during walking were analyzed by the open-source program OPENSIM [24-26] using an experimental data set of GIL02. The data set contains the walking kinematics and dynamics of a 14.6-year-old female of weight $66 \mathrm{~kg}$, with legs $0.9 \mathrm{~m}$ long. The inverse dynamics analysis suggested that during human walking at a speed of $5.5 \mathrm{~km} / \mathrm{h}$ the maximum angular velocity of the knee motion is about $400 \mathrm{deg} / \mathrm{s}$. To support human walking at the same speed, an actuator module of the body support device should 
be capable of producing a continuous torque at a minimum of $38 \mathrm{Nm}$, assuming that the wearer weighs $85 \mathrm{~kg}$.

\section{Design Concept}

This section explores the design concepts of kinematic structure and actuation system to develop the first version of the Knee-Assistive Robotic Exoskeleton (KARE-1), a body support mechanism. In addition to the basic requirement of providing a proper physical support to a human body, a body support mechanism should be lightweight and driven by small actuators to guarantee its applicability to industrial sites. A novel multi-body linkage system that makes use of a passive element and takes advantages of singular configuration was considered to meet the requirements.

\subsection{Singularities}

In the previous study twelve four-bar linkage designs are investigated, each of which has a single passive element and a single active element ([20]). Designs with different choice of either rotary or linear for the passive and single element, were evaluated if they satisfy the main design guidelines mentioned above. And, the mechanism with a rotary active element and a linear passive element was found to have advantages of high torque in/output efficiency and torque production capacity. In this work such mechanism was further developed to relieve the knee torque loadings during sitting and to implement high backdrivability when walking in the following ways:

1. During static sitting posture, the body support device is controlled to take a singular configuration of triangular shape at which the spring force would be equivalent to the body weight. Since two forces are in equilibrium, there is no muscle force from a human body and no active torque by an actuator. This mode is called the Fully Supportive Singular Configuration (FSSC, Figure 2a).

2. When a human body is in transition mode, the body support can assume a configuration so that the spring force would be a certain percentage more than the static due. This mode is called the Assistive Quasi-Singular Configuration (AQSC, Figure 2b). During this mode the body support needs an actuator torque to produce the necessary spring force. The amount of actuator torque is described in Section 3.2.

3. In walking motion, the body support assumes a singular configuration such that the spring element remains uncompressed and produces zero resistance on knee motion. This mode is called the walking singularity configuration (WSC, Figure 2c). Table 2 shows the features of the designed device.

Table 2. The three representative phases causing muscle fatigue, and features of the proposed device.

\begin{tabular}{cccc}
\hline Category. & Standing & Saddle Stance/Deep Sitting & Walking \\
\hline Assistance Function & Not applicable & Full support or partial assist & Not applicable \\
Method for Assistance & Not applicable & Full spring force or desired spring force & Not applicable \\
Power Consumption & Not applicable & No or economy & Not applicable \\
Fatigue Reduction & Not effective & Improved & Not good (extra device mass) \\
\hline
\end{tabular}




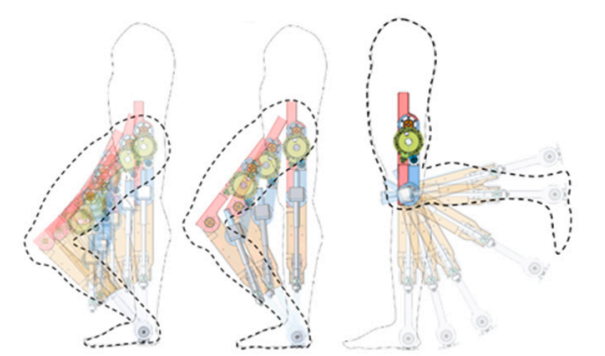

(a)

(b)

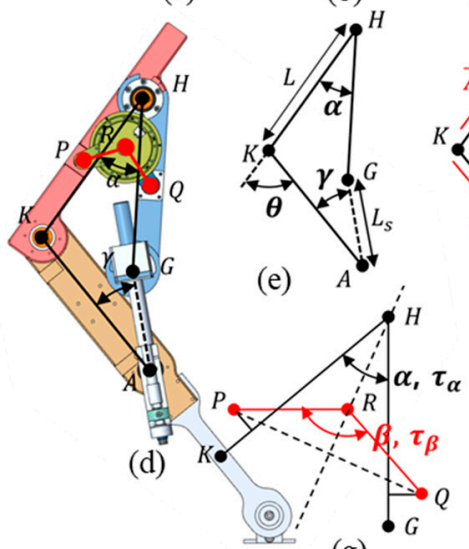

(g) (c)

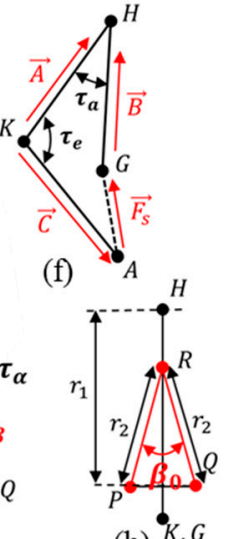

(h) $K, G$

Figure 2. Representative motions and mechanisms. (a) Motion in Fully Supportive Singular Configuration; (b) Assistive Quasi-Singular Configuration; (c) Walking Singular Configuration. Schematic of proposed mechanism (d-h): (d) 3D CAD model; (e) Kinematic definition of proposed mechanism; (f) Free body diagram of proposed mechanism. Extended link mechanism for extra reduction ratio $(\mathbf{g}, \mathbf{h})$.

\subsection{Basic Kinematics and Kinetics}

A four-bar linkage system with an active element of a rotary actuator and a passive element of a linear spring was proposed for KARE-1 (Figure 2). Three linkages $(\overline{\mathrm{HK}}, \overline{\mathrm{KA}}$, and $\overline{\mathrm{HG}})$ are of equal lengths, $L$. The knee joint angle $\theta$ is one between $\overline{\mathrm{HK}}$ and $\overline{\mathrm{KA}}$. A passive joint angle $\gamma$ is one between $\overline{\mathrm{KA}}$ and $\overline{\mathrm{GA}}$. The remaining linkage $(\overline{\mathrm{GA}})$ has a slide section along which joint $\mathrm{G}$ moves. When the slide moves, the length of the linear spring $L_{S}$ varies accordingly. The active angle $\alpha=\angle K H G$ is controlled by the actuator system at joint $\mathrm{R}$.

From the triangle GHA, the law of cosine gives the relation of:

$$
\alpha=\frac{\pi}{2}-\cos ^{-1}\left(\frac{L^{2}+4 L^{2} \cos ^{2}\left(\frac{\theta}{2}\right)-L_{s}^{2}}{4 L^{2} \cos \left(\frac{\theta}{2}\right)}\right)
$$

From the triangle GAH, the law of cosine gives the relation of:

$$
\gamma=\frac{\pi}{2}-\cos ^{-1}\left(\frac{L S^{2}+4 L^{2} \cos ^{2}\left(\frac{\theta}{2}\right)-L^{2}}{4 L_{S} L \cos \left(\frac{\theta}{2}\right)}\right)
$$

Spring force $F_{S}$ is a function of spring length $L_{s}$. An elastic torque, a knee torque that is generated by $F_{s}$, is denoted as $\tau_{e}$ in Equation (3).

$$
\tau_{e}=L \sin (\gamma) F_{s}\left(L_{s}\right)
$$

A gravity torque, a knee torque that is generated by a weight force $W$, is denoted as $\tau_{g}$ in Equation (4). $W$ is a summed weight force of human body parts above the knee and the KARE-1's 
upper linkage parts. It is assumed that $W$ passes the center of the upper linkage $\overline{\mathrm{HK}}$ since the upper body usually bends forward when sitting down to keep stability.

$$
\tau_{g}=\frac{w}{2} L \sin \left(\frac{\theta}{2}\right)
$$

The body assistance ratio $\mathfrak{R}$ is defined as the $\tau_{e}$ divided by the $\tau_{g}$ :

$$
\mathfrak{R}=\tau_{e} / \tau_{g}
$$

Plugging in Equations (3) and (4) into (5) gives:

$$
L_{s}=F_{s}^{-1}\left(\frac{W \sin \left(\frac{\theta}{2}\right)}{2 \sin (\gamma)} \mathfrak{R}\right)
$$

Plugging in Equation (6) into (2) gives a nonlinear equation in terms of $\gamma, \theta$, and $\mathfrak{R}$.

$$
\gamma=\frac{\pi}{2}-\cos ^{-1}\left(\frac{\left\{F_{s}^{-1}\left(\frac{W \sin \left(\frac{\theta}{2}\right)}{2 \sin (\gamma)} \mathfrak{R}\right)\right\}^{2}+4 L^{2} \cos ^{2}\left(\frac{\theta}{2}\right)-L^{2}}{4\left\{F_{s}^{-1}\left(\frac{W \sin \left(\frac{\theta}{2}\right)}{2 \sin (\gamma)} \mathfrak{R}\right)\right\} L \cos \left(\frac{\theta}{2}\right)}\right)
$$

Then, unknown $\gamma$ is solved in terms of $\theta$ and $\mathfrak{R}$ numerically, even if not explicitly. Then, $L_{s}$ and $\alpha$ are accordingly solved when plugging $\gamma$ into Equations (6) and (2).

An active torque $\tau_{a}$ at joint $\mathrm{H}$, needed to keep the static balance with the spring and gravity forces, is defined as follows:

$$
\tau_{a}=L \sin (\theta-\alpha-\gamma) F_{s}\left(L_{s}\right)
$$

Once again, $\tau_{a}$ can be expressed in terms of $\theta$ and $\mathfrak{R}$. The $\tau_{a}$ is produced by an actuator and thus is a unique element that consumes energy. It means that the behavior of $\tau_{a}$ would determine the energy consumption of the proposed body support.

\subsection{Mode-Specific Kinematics and Kinetics}

Figure 2 a shows the FSSC mode when joint $\mathrm{G}$ stays on the line $\overline{\mathrm{HA}}$ and $\alpha$ is equal to $\theta / 2$. Figure $3 a$ is a contour plot of $\Re$ as a function of $\theta$ and $\alpha$. Any point on the diagonal line of Figure 3a represents the FSSC configuration, and the body assistance ratio $\mathfrak{R}$ is equal to one. In this case, the spring force statically balances with gravity, allowing the wearer to sit without knee muscle force.

Figure 2c shows the WSC mode when joint G of the KARE- 1 stays at joint $\mathrm{K}$ and $\alpha$ is equal to zero. At any point on the horizontal axis of Figure 3a corresponding to transition from standing ( $\theta=0$ degrees) to sitting ( $\theta=90$ degrees) during the WSC, the body assistance ratio and spring force are both zero, and the spring is uncompressed. Thus, leg movements encounter minimal resistance from the KARE-1.

Figure $2 \mathrm{~b}$ shows the AQSC mode when joint $\mathrm{G}$ stays between line $\overline{\mathrm{HA}}$ and joint $\mathrm{K}$ and $\alpha$ is between 0 and $\theta / 2$. As shown in Figure $3 \mathrm{a}$ an actuator torque is non-zero in the mode. A peak value of required actuator torque during the AQSC is quite high near the gray-colored zone in Figure $3 b$, but the actuator capacity does not have to satisfy the peak value since the actuator needs to produce the peak value just temporarily when the body support device transitions from one singularity region to another. Figure $3 c$,d shows the exerted torque and electric power loss as functions of the knee angle. 


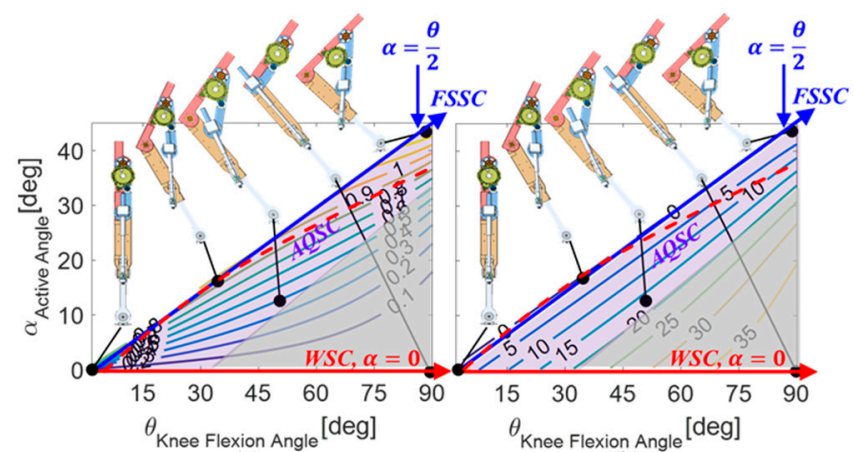

(a)

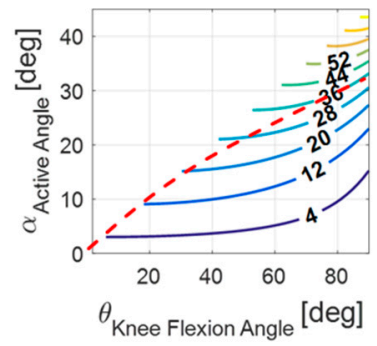

(c)

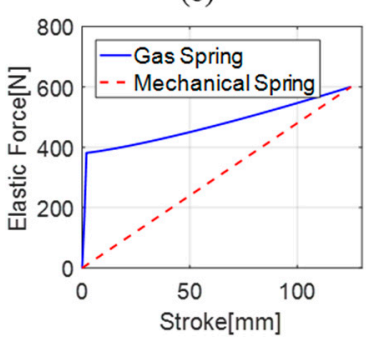

(d)

Figure 3. Kinetic analysis of the KARE-1. Contour of body assistance ratio $\mathfrak{R}$ as a function of $\theta$ and $\alpha$ (a). Contour of active torque $\tau_{a}$ as a function of $\theta$ and $\alpha(\mathbf{b})$. Contour of an elastic torque $\tau_{e} \mathfrak{R}$ as a function of $\theta$ and $\alpha$ (c). Comparison of gas and mechanical spring forces with respect to a stroke (d). Red dash curves in $(\mathbf{a}-\mathbf{c})$ divide the $\theta$ and $\alpha$ domain with $R$ at 0.8 . Purple areas in $(\mathbf{a}, \mathbf{b})$ stand for the $\theta$ and $\alpha$ domain with $\tau_{a}$ below 20 .

\subsection{Extended Link Mechanism}

To minimize the size of actuator, while still meeting the output torque requirement, extended linkages $\overline{\mathrm{PR}}$ and $\overline{\mathrm{RQ}}$ were added with a rotary actuator installed to these linkages (Figure $2 \mathrm{~d}-\mathrm{h}$ ). The extended linkages amplify the torque output of the actuator by the reduction ratio $N$ of Equation (9), which is defined as the ratio of change in an actuator's rotation angle $\angle \mathrm{PRQ}(=\beta)$ to that of the active angle $\angle \mathrm{KHG}(=\alpha)$ :

$$
\begin{gathered}
N=\frac{d \beta}{d \alpha}=\frac{\mu\left(-\tan \frac{\beta_{0}}{2} \sin \frac{\alpha}{2}+\cos \frac{\alpha}{2}\right)}{\cos \left(\sin ^{-1}\left(\mu\left(\tan \frac{\beta_{0}}{2} \cos \frac{\alpha}{2}+\sin \frac{\alpha}{2}\right)\right)\right)} \\
\tau_{b}=N \tau_{a},
\end{gathered}
$$

where the design variable is $\mu \equiv r_{1} / r_{2}, r_{1}$ is the minimum distance between point $P$ and $\overline{\mathrm{HK}}$, and $r_{2}$ is the length of $\overline{\mathrm{RQ}}$. $\beta_{0}$ is the angle of $\angle \mathrm{PRQ}$ when the $\alpha$ is zero, and is set to be 17 degrees to consider the thickness of the link and the size of the bearing. These design parameters are summarized in Table 3. Finally, $\tau_{b}$ in Equation (10) is the output torque transmitted to the center $\mathrm{R}$ of the extended link mechanism from the actuator.

Table 3. Kinematics Parameters.

\begin{tabular}{ccccc}
\hline $\boldsymbol{L}$ & $\boldsymbol{\alpha}_{0}$ & $\boldsymbol{\beta}_{0}$ & $\boldsymbol{r}_{1}$ & $\boldsymbol{r}_{2}$ \\
\hline $0.2 \mathrm{~m}$ & $34 \mathrm{deg}$ & $17 \mathrm{deg}$ & $100 \mathrm{~mm}$ & $51 \mathrm{~mm}$ \\
\hline
\end{tabular}

The simulations in Figure 4a,b were performed to show the effect of the extended linkage mechanism. Parameters in Table 4 were chosen with reference to previous work $[21,27]$. Their results 
show the relation between the speed-torque and generated output when each mechanism of Figure $4 a, b$ was applied to the simple human model in Figure $4 \mathrm{c}$,d. The final design with the extended linkages could achieve reduction in the stall torque requirement of the rotary actuator by $30 \%$, relative to the model without it.

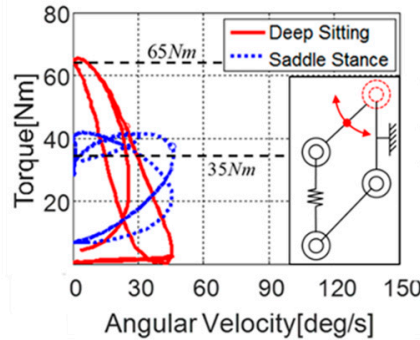

(a)

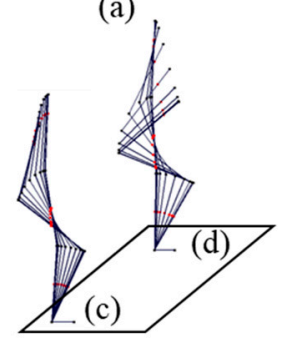

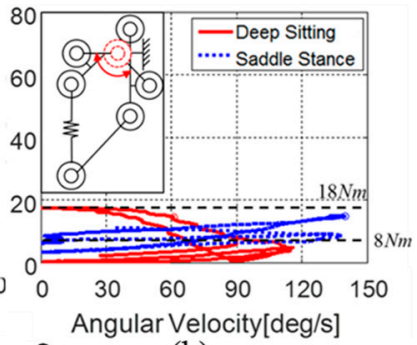

(b)

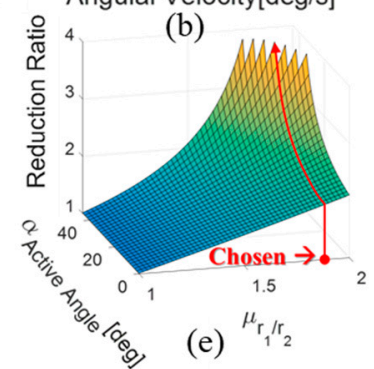

Figure 4. Simulation of standing-to-sitting motion, and vice versa. Simulation result on torque-angular velocity curve computed for knee joint actuation without (a) and with (b) the extended link mechanism, animated trajectory of saddle stance (c) and deep sitting. (d) Contour plot of the additional reduction ratio created by the extended link mechanism with respect to the active angle and design variable (e).

Table 4. A Simple 4-Link Model for Simulation.

\begin{tabular}{ccccc}
\hline & Torso & Thigh & Shank & Foot \\
\hline Mass & $25 \mathrm{~kg}$ & $7.5 \mathrm{~kg}$ & $4.5 \mathrm{~kg}$ & $3 \mathrm{~kg}$ \\
Length & $0.90 \mathrm{~m}$ & $0.43 \mathrm{~m}$ & $0.42 \mathrm{~m}$ & - \\
COM & $0.45 \mathrm{~m}$ & $0.22 \mathrm{~m}$ & $0.21 \mathrm{~m}$ & - \\
\hline
\end{tabular}

The contours of the reduction ratio with respect to the $\mu$ and the $\alpha$ are plotted in Figure 4e. While the $N$ increases with the $\mu$, it is limited around singularities when the $\alpha>40$ degrees and/or $\mu>1.9$ (Figure 4e). When the $\mu$ is set to 1.9 , the $N$ increases monotonically from 2 to 10 with the $\alpha$.

\subsection{Choice of Actuator Type}

A special type of spring is chosen as a passive element for the KARE-1 based on the following observations. When the KARE-1 is in the FSSC configuration, the spring force should be equal to the body weight. Since this body weight is constant over different sitting heights, the spring force should be also constant. While a usual type of spring would change force production as sitting height changes, a preloaded gas spring shows small variation over a wide range change in its stroke, as in Figure $3 \mathrm{~b}$. Also, a sudden drop in force production at zero stroke is also beneficial to remove interference with leg motion during walking.

The spring force is approximated by:

$$
F_{S}\left(L_{S}\right)=\left\{\begin{array}{cl}
F_{o}+k\left(L-L_{S}\right)^{n} & \left(\varepsilon L>L_{S}\right) \\
{\left[F_{o}+k *\left(L-L_{S}\right)^{n}\right] *\left[\frac{L-L_{S}}{L-L * \varepsilon}\right]^{n}} & \left(\varepsilon L \leq L_{S}<L\right) \\
0 & \left(L \leq L_{S}\right)
\end{array}\right.
$$


where $F_{o}=380 \mathrm{~N}$ is the preloaded spring force, $k=2960 \mathrm{~N} / \mathrm{m}$ is a nonlinear spring constant, and $n=1.25$ is the power constant with which the output spring force best fits experimental results. An additional constant, $\varepsilon=0.99$, defines the stroke length at which the spring force starts to build abruptly from an uncompressed state.

\section{Controller Design}

The overall hardware setup of the KARE-1 is shown in Figure 5. It has a main control system based on an STM32 micro-controller that operates at $100 \mathrm{~Hz}$ in real time. It has a communication module based on Bluetooth technology between the monitoring tablet device and the main control system.

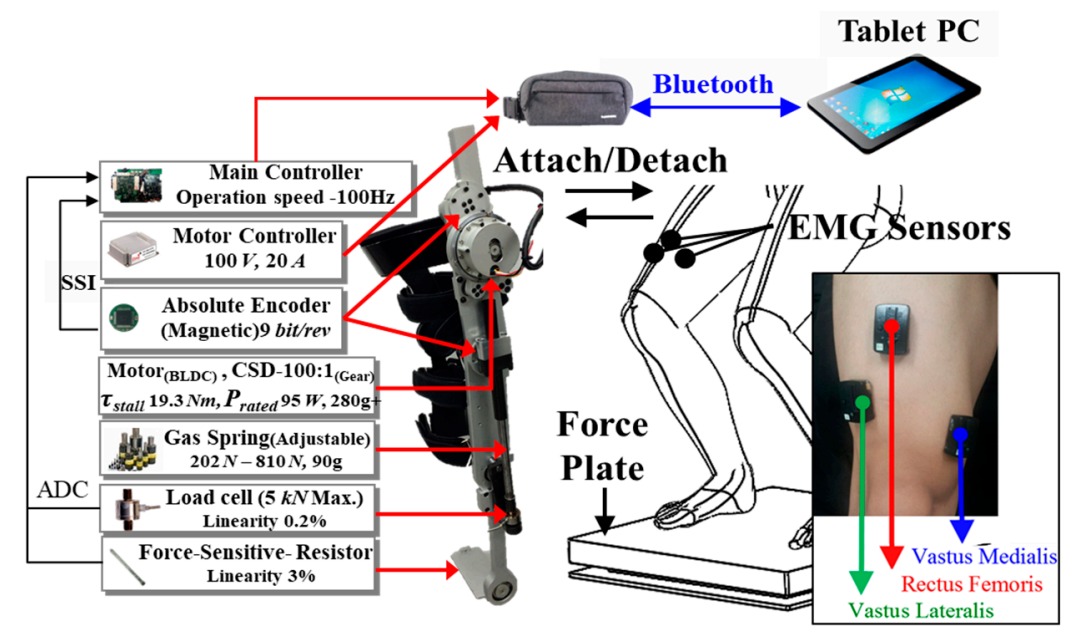

Figure 5. Hardware setup of KARE-1.

\subsection{Mode Switch}

Figure 6 illustrates the control algorithm of the KARE-1. As mentioned in the previous section there are three basic modes for the KARE-1 control. The controller automatically detects walking through the foot pressure and knee kinematic sensors, and the KARE-1 is controlled in the WSC mode. When the KARE-1 is not in the WSC mode, it switches to either AQSC or FSSC mode according to the pre-selection by a user. Transition of control mode between the FSSC and the WSC, or between the AQSC and the WSC, is determined by two conditions. When foot pressure from the force-resistive sensors (FSR) is zero or the angular velocity of the knee $\dot{\theta}$ is greater than a trigger value $S_{t}$, the control of the KARE- 1 is in WSC mode and the active angle is set to be zero. The $S_{t}$ is set to $15 \mathrm{deg} / \mathrm{s} \mathrm{by} \mathrm{trial}$ and error.

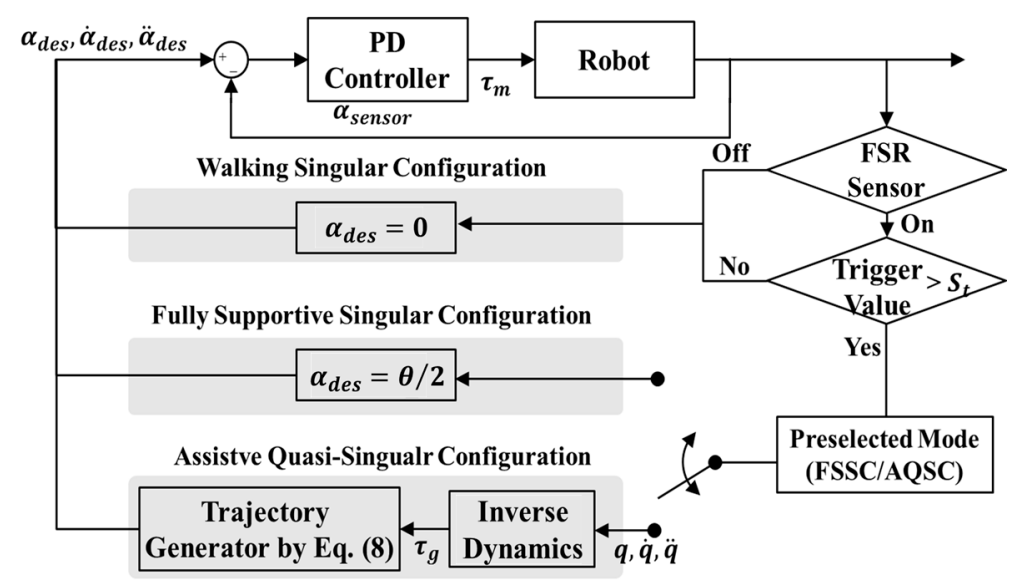

Figure 6. Control diagram of KARE-1. 


\subsection{Control Strategy}

As in Section 3.4 the desired active angle $\alpha_{\text {des }}$ is controlled to be $\theta / 2$ in the FSSC mode, and is controlled to be zero in the WSC mode, which is done by a displacement control. In AQSC mode the KARE-1 is basically driven by a PD control, Equation (12), in which the desired value of $\alpha$ as well as the driving torque $\tau_{a}$ is calculated by the body assistance ratio $\mathfrak{R}$. Based on the two values and state variables of the KARE-1, the desired actuator torque $\tau_{m}$ is calculated by Equation (13).

$$
\begin{gathered}
\tau_{a}+N \tau_{m}=J \ddot{\alpha}+b \dot{\alpha}+k \alpha \\
\tau_{m}=N^{-1}\left(J Q+b \dot{\alpha}+k \alpha-\tau_{a}\right) \\
Q=\ddot{\alpha}_{d e s}+K_{v}\left(\dot{\alpha}_{d e s}-\dot{\alpha}\right)+K_{p}\left(\alpha_{\text {des }}-\alpha\right)
\end{gathered}
$$

where $J$ is the rotational inertia of a linkage $\overline{H G} ; \mathrm{b}$ and $\mathrm{k}$ are respectively the viscous friction and stiffness coefficient of joint $\mathrm{H}$. Control gains for $\mathrm{K}_{\mathrm{p}}$ and $\mathrm{K}_{\mathrm{v}}$ are tuned through the critical damping condition using error dynamics; $\dot{\alpha}_{d e s}, \ddot{\alpha}_{d e s}$ are determined from a given time function of $\alpha_{d e s}$ using the polynomial spline technique.

\section{Prototype Test}

To verify the performance of the KARE-1, two sets of experimental tests were carried out on ten human subjects whose anthropometric metrics are listed in Table 5.

Table 5. Information of Subjects.

\begin{tabular}{c|c|c|c|c|c|c|c|c|c|c}
\hline & $\mathbf{1}$ & $\mathbf{2}$ & $\mathbf{3}$ & $\mathbf{4}$ & $\mathbf{5}$ & $\mathbf{6}$ & $\mathbf{7}$ & $\mathbf{8}$ & $\mathbf{9}$ & $\mathbf{1 0}$ \\
\hline Weight(kg) & 75 & 58 & 71 & 61 & 74 & 69 & 85 & 84 & 68 & 62 \\
Age & 25 & 23 & 25 & 25 & 25 & 27 & 30 & 29 & 27 & 27 \\
Height(cm) & 168 & 173 & 174 & 170 & 179 & 171 & 170 & 180 & 175 & 168 \\
\hline
\end{tabular}

In the first set of tests a human subject equipped with the body support device was instructed to walk at the speed of $4 \mathrm{~km} / \mathrm{h}$ along a straight lane of $20 \mathrm{~m}$ length. A human subject adjusted walking speed using the markers on the lane that were placed every $1 \mathrm{~m}$ along the lane and a ticking sound from a metronome. A human subject made two round trips of walking for each case, with and without the KARE-1.

In the second test, a human subject was instructed to do ten repetitions of the stand-sit-stand motion. One repetition is made up of four segments, i.e., stand-to-sit (3 s), keep sitting (2 s), sit-to-stand $(3 \mathrm{~s})$, and standing ( $2 \mathrm{~s})$. During the stand-sit-stand test, $\mathfrak{R}$ of the KARE- 1 is set to a constant value out of $0 \%, 40 \%, 70 \%$, and $100 \%$ to observe its effect on the muscle loadings. The snapshots of a human user during the second and the third tests are presented in Figure 7.

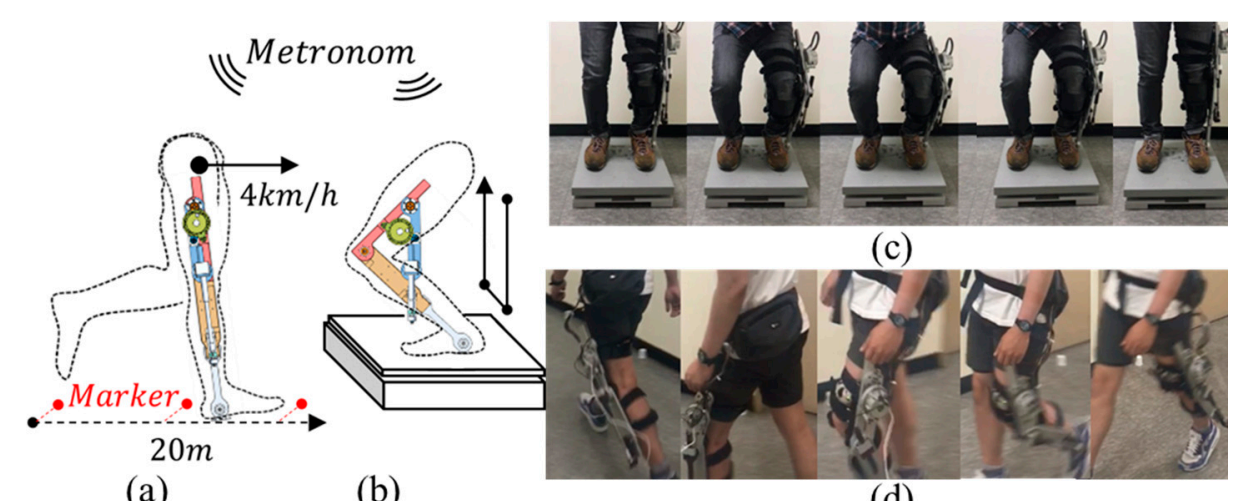

(a)

(b)

(d)

Figure 7. Human subject test conditions. Experimental setup $(\mathbf{a}, \mathbf{b})$ and snapshots $(\mathbf{c}, \mathbf{d})$. 
The output kinematics of the KARE-1 show that the actuating linkage ( $\overline{\mathrm{HG}}$ of Figure 2$)$ was successfully controlled in the WSC mode during the walking test and in the AQSC mode during the stand-sit-stand test as in Figure 7c,d. Figure 8 shows that the KARE-1 successfully produced the output actuator torque with the consumed power loss as desired.

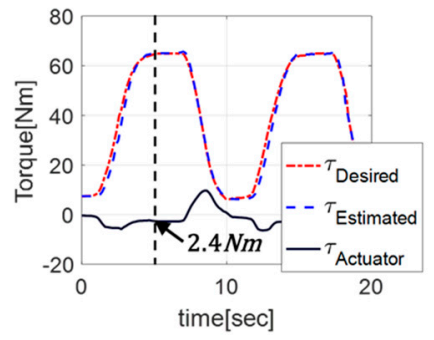

(a)

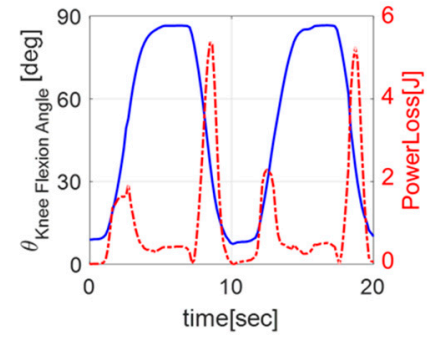

(b)

Figure 8. Power consumption of the KARE-1. Estimated knee joint torques and actuator torque after gearbox application (a), knee joint angle and electrical power loss (b).

Because the KARE-1 has been worn on the left leg only, a human subject may lean on the left side or the right and unexpected changes in muscle activities may occur. To avoid this situation, the human subjects were asked to keep their balance during the tests. During the tests, the center of pressure (CoP) of the ground reaction forces were recorded during stand-sit-stand test. In Figure $9 \mathrm{a}-\mathrm{c}$, the trajectories of the CoP during stand-sit-stand (STS) movement are compared against the case without the KARE-1. As shown, the CoP of the foot moved slightly towards the back and left, but the range of movement was small enough to say that the additional mass of the KARE-1 barely affected the balance.

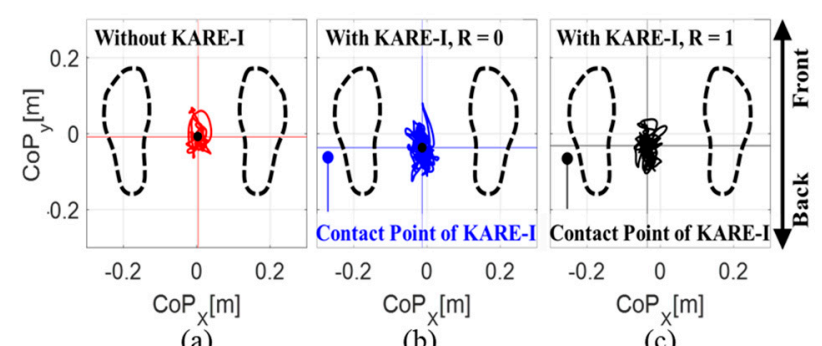

(a)

(b)

(c)

Figure 9. Trajectories of the center of pressure $(\mathrm{COP})$ measured by the force plate during the stand-sit-stand motion: without KARE-1 (a), with KARE-1, $\mathfrak{R}=0$ (b), and with KARE-1, $\mathfrak{R}=1$ (c).

To evaluate the reduction in muscle loading by the KARE-1, the activation levels of the three major knee extensors were measured using an electromyography (EMG) sensor. Surface EMG signals of the vastus lateralis, vastus medialis, and rectus femoris were collected using a Trigno ${ }^{\mathrm{TM}}$ from DELSYS, Ltd. Natick, MA, USA. Additional tests were conducted to collect EMG data during the maximum voluntary contraction (MVC) according to the procedures suggested in previous work [28]. The locations of EMG electrodes are shown in Figure 5. The EMG signals were collected at $4000 \mathrm{~Hz}$, offset-cancelled, rectified, and band filtered to retain signals between 50 and $200 \mathrm{~Hz}$ using the fourth-order Butterworth filter. The processed EMGs were again rectified and low-pass filtered with a cutoff frequency of $20 \mathrm{~Hz}$ using the second Butterworth filter. They were then averaged and normalized by EMGs at MVC.

The muscle activations of knee extensors decreased as the body assistance ratio increased during stand-sit-stand (Figure 10b), as expected. However, there were still non-zero activations even at 100\% of body assistance ratio. This accounts for the torque tracking error so that muscle forces need to compensate for the discrepancy between the gravity torque and the elastic torque, or for co-contraction of both knee flexors and extensors to stabilize the knee joint. 


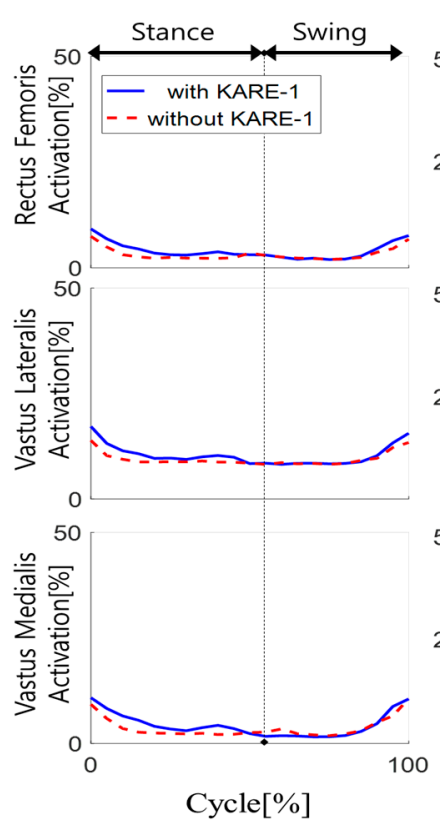

(a)

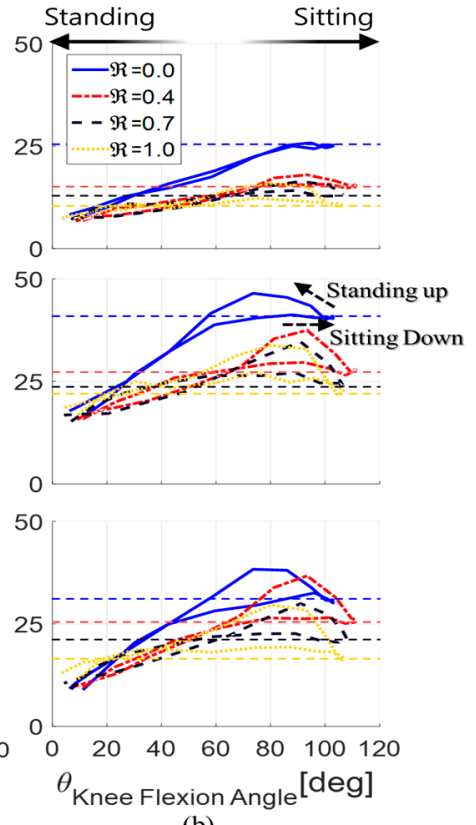

(b)

Figure 10. Normalized muscle activations of major knee extensors compared: walking with and without the KARE-1 (a), stand-sit-stand motions with different body assistance ratio (b).

Muscle activation during stand-up was recorded as higher than for sit-down. Though more torque is required to push up the body mass against gravity during stand-up than sit-down, the KARE-1 in the AQSC mode provides the same level of support torque for both motions. Thus, the knee extensor muscles are activated more to compensate for the torque difference. During walking at $4 \mathrm{~km} / \mathrm{h}$, overall muscle activation was observed to be higher with the KARE-1 than without it for both stance and swing phase (Figure 10a). This is because the inertial forces from a $3 \mathrm{~kg}$ mass of the KARE- 1 and friction forces from the bearing at the linkage joints would have imposed an extra loading on the knee muscles.

The actuator torque was estimated from the current induced in the actuator using electromagnetic relationships. The elastic torque and the gravity torque were calculated from the spring force and the knee kinematics measured from the encoder. Based on the measured current of the motor, power consumption was estimated from electrical energy loss due to the resistance of the electric motor.

\section{Discussion}

The practical applicability of the KARE-1 to the workplace has been reviewed from two standpoints. One is the relative muscle efforts of a human worker and the other is the absolute energy consumption of the KARE-1. For the review, two metrics that quantify the two concepts are defined and evaluated based on the experimental data of the previous tests.

\subsection{Battery Capacity}

The output actuator torque was estimated based on the induced current in the actuator. Figure 8b shows a time trajectory of the electrical power loss based on the relationship in Equation (15):

$$
\text { Electrical Power loss }=\left(\tau_{m} / N k_{t}\right)^{2} R_{m}
$$

where a torque constant $k_{t}$ is 0.0407 , a gear ratio $N$ is 100 , and electric resistance $R_{m}$ is 0.814 .

Based on the electrical power consumption of the actuator during individual tasks, the battery capacity requirement is calculated for scenario $A$ of working conditions in Section 2 that includes $40 \%$ of walking phase (24 s) and 60\% sitting phase (36 s). More specifically, the sitting phase included stand-to-sit for $3 \mathrm{~s}$, sitting for $30 \mathrm{~s}$, and sit-to-stand for $3 \mathrm{~s}$. The energy consumption during the scenario 
was estimated from the experimental data and was calculated to be approximately $0.5 \mathrm{Wh}$. Energy consumed by the control board and sensors was not included in the calculation. Based on the energy density of recent batteries $(100 \mathrm{Wh} / \mathrm{kg})$, the proposed KARE-1 could operate for over eight hours with a battery mass of only $100 \mathrm{~g}$.

\subsection{Muscle Efforts}

Muscle effort has been defined based on muscle activation during two activities. $E$ is the normalized EMG signals to the MVC signals. $E_{w}$ and $E_{S}$ denote the $E$ measured during walking and sitting, respectively, acquired from the results of Figure 10.

$$
F=\int_{0}^{60-t_{s}}\left(E_{w}\right) d \tau+\int_{0}^{t_{s}}\left(E_{s}\right) d \tau
$$

The relative muscle effort with the KARE-1 compared to the case without it is defined as $G$ in Equation (17).

$$
G=F^{W} / F^{U}
$$

where the superscripts ' $W$ ' and ' $U$ ' stand for the case with the KARE-1 and the case without assistance, respectively. It is shown that $\mathrm{G}$ drops down, or the KARE-1 becomes more efficient, as the period of sitting duration increases. The $G$ value drops when the body assistance ratio $\mathfrak{R}$ increases. However, as the period of walking time increases, the efficiency drops and $G$ becomes more than one (see the gray-colored circle, Figure 11), which means that the KARE-1 is not efficient anymore.

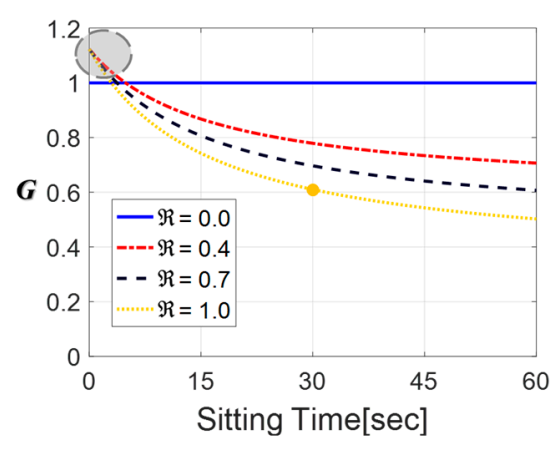

Figure 11. A contour plot of effectiveness factor $G$ with various body assistance ratios $\mathfrak{R}$ from 0 to 1 . (Walking speed is set at $4 \mathrm{~km} / \mathrm{h}$ ).

While $G$ gives a rough estimation on how the efficiency of the KARE-1 with respect to the body assistance ratio and task ratio, it has limitations in that: (1) the estimation is based on the extrapolation of measured data, and (2) changes of muscle by adaptation or fatigue are not considered.

Finally, the performance specifications of the proposed assistive robotic exoskeleton are summarized and compared with top commercial products in Table 6. This verifies the feasibility and validity of the proposed exoskeleton.

Table 6. Comparison with Commercial Knee-Assistive Devices.

\begin{tabular}{cccc}
\hline- & Noonee [3] (Early Type) & BWAS [5] & KARE-1 \\
\hline Actuation & Passive & Active & Active \\
Assist Capacity & Full support/100 kg/leg & Partial Assist $/ 10 \mathrm{~kg} / \mathrm{leg}$ & Full support/42.5 kg/leg \\
Weight & $1 \mathrm{~kg} / \mathrm{leg}$ (Target) & $3.25 \mathrm{~kg} / \mathrm{leg}$ & $3.0 \mathrm{~kg} / \mathrm{leg}$ \\
Mechanism & Damper and Locking System & $1 \mathrm{Motor} / \mathrm{leg}$ & $1 \mathrm{Motor} / \mathrm{Leg}$ \\
Operation time & $8 \mathrm{~h}$ & $2 \mathrm{~h}$ & Over $8 \mathrm{~h}$ \\
\hline
\end{tabular}




\subsection{Practical Issues}

Since the KARE-1 has been developed and tested only in a laboratory environment, there are more design considerations for practical use than described so far. While electricity is provided through an external power cable in the laboratory, one needs to carry a battery pack in the field. Thus, design issues on where to carry the battery would arise. When not carried next to the human body, it would act like a wobbling mass and cost muscle effort to overcome its dynamic forces. When carried close to a human body, its heat emission may cause fatigue and discomfort.

Since there no direct transfer of the robotic device's weight to the ground, the ankle is loaded consistently, which may lead to orthopedic disorder after long term use. Such weight force causes a robotic device to slide down, and one should tie the linkages to the thigh and the shank or hang the upper linkage through extra straps from the waist.

\section{Conclusions}

In this paper, a robotic device was proposed for supporting a worker's body weight. Since the body support device was tightly attached to the wearer's leg, it had to be actuated naturally in accordance with the user's intention to move. An exoskeleton design based on a four-bar mechanism with two actuators, one active and one passive, was developed for mobility and lower energy consumption.

The proposed mechanism shows potential for presenting an energy-efficient assistive robot in multiple common configurations. By making use of these singular configurations as well as the passive element, the proposed design can achieve mechanical back-drivability while walking and mechanical spring-based force amplification during body support phases. In the active mode, it can provide body support torque at any knee posture with less energy expenditure using actuator torque along with spring force. The performance of the proposed body assistive device was verified through extensive simulations and human testing. Energy consumption based on these results showed that the proposed body support is applicable to industrial practices.

However, for industry applications the following issues remain unresolved: only the knee flexion-extension movement was considered in this work, and the exoskeleton was attached to the side of the lower limb. These bring about a certain discrepancy from natural distributions in both mass and motion at the knee joint [29], and undesirable yawing torques at the hip while walking, which restrain the other degrees of freedom of the leg. Hence, further development of the proposed design or a foot mechanism may be required to relieve these discomforts. Wearability is another issue. Due to tight contact with the thigh and the shank of the human body, the body support device can cause discomfort when used over a number of hours. Thus, in further research, the authors will try to solve these difficulties by introducing an advanced human-robot interactive control method.

Author Contributions: Conceptualization, J.N. and W.Y.; methodology, H.J.K. and J.N.; validation, J.N. and H.J.K. and W.Y.; formal analysis, J.N.; investigation, J.N. and H.J.K.; data curation, J.N. and H.J.K.; writing一original draft preparation, H.J.K. and J.N.; writing—review and editing, H.J.K. and W.Y.; project administration, W.Y. All authors have read and agreed to the published version of the manuscript.

Funding: This research was supported by the Basic Science Research Program through the National Research Foundation of Korea (NRF), funded by the Ministry of Education (2017R1A2B3010336).

Acknowledgments: The work reported in this paper was conducted during the sabbatical year of Kwangwoon University in 2019.

Conflicts of Interest: The authors declare no conflict of interest with respect to the research, authorship, and/ or publication of this article. 


\section{References}

1. Chu, A.; Kazerooni, H.; Zoss, A. On the biomimetic design of the Berkley Lower Extremity Exoskeleton (BLEEX). In Proceedings of the 2005 IEEE International Conference on Robotics and Automation, Barcelona, Spain, 18-22 April 2005; pp. 4345-4352.

2. Industrial Human Augmentation System (iHAS). Available online: https://www.ncms.org/ (accessed on 18 July 2020).

3. Noonee. Available online: https://www.noonee.com/ (accessed on 18 July 2020).

4. Robo-Mate. Available online: http://www.robo-mate.eu/ (accessed on 18 July 2020).

5. Ikeuchi, Y.; Ashihara, J.; Hiki, Y.; Kudoh, H.; Noda, T. Walking assist device with bodyweight support system. In Proceedings of the 2009 IEEE/RSJ International Conference on Intelligent Robots and Systems, St. Louis, MO, USA, 10-15 October 2009; pp. 4073-4079.

6. Kawamoto, H.; Sankai, Y. Comfortable power assist control method for walking aid by HAL-3. In Proceedings of the IEEE International Conference on Systems, Man and Cybernetics, Yasmine Hammamet, Tunisia, 6-9 October 2002; Volume 4, pp. 1-6.

7. Vanderborght, B.; Albu-Schäffer, A.; Bicchi, A.; Burdet, E.; Caldwell, D.G.; Carloni, R.; Catalano, M.G.; Eiberger, O.; Friedl, W.; Ganesh, G.; et al. Variable impedance actuators: A review. Robot. Auton. Syst. 2013, 61, 1601-1614. [CrossRef]

8. Ham, V.R.; Sugar, T.G.; Vanderborght, B.; Hollander, K.W.; Lefeber, D. Compliant actuator designs: Review of actuators with passive adjustable compliance/controllable stiffness for robotic applications. IEEE Robot. Autom. Mag. 2009, 16, 81-94. [CrossRef]

9. Paine, N.; Oh, S.; Sentis, L. Design and control considerations for high-performance series elastic actuators. IEEE/ASME Trans. Mech. 2014, 19, 1080-1091. [CrossRef]

10. Veneman, J.F. A series elastic- and Bowden cable-based actuation system for use as torque actuator in exoskeleton-type robots. Int. J. Robot. Res. 2006, 25, 261-281. [CrossRef]

11. Beyl, P. Design and Control of a Knee Exoskeleton Powered by Pleated Pneumatic Artificial Muscles for Robot-Assisted Gait Rehabilitation Chair. Ph.D. Thesis, Vrije Universiteit Brussel, Brussels, Belgium, 2010.

12. Tondu, B. A seven-degree-of-freedom robot-arm driven by pneumatic artificial muscles for humanoid robots. Int. J. Robot. Res. 2005, 24, 257-274. [CrossRef]

13. Wolf, S. New variable stiffness design: Maching requirements of the next robot generation. In Proceedings of the 2008 IEEE International Conference on Robotics and Automation, Pasadena, CA, USA, 19-23 May 2008; pp. 1741-1746.

14. Tsagarakis, N.G.; Sardellitti, I.; Caldwell, D.G. A new variable stiffness actuator (CompAct-VSA): Design and modelling. In Proceedings of the 2011 IEEE/RSJ International Conference on Intelligent Robots and Systems, San Francisco, CA, USA, 25-30 September 2011; pp. 378-383.

15. Chen, J.Z.; Liao, W.H. Design, testing and control of a magnetorheological actuator for assistive knee braces. Smart Mater. Struct. 2010, 19, 035029. [CrossRef]

16. Nikitczuk, J.; Weinberg, B.; Mavroidis, C. Rehabilitative knee orthosis driven by electro-rheological fluid based actuators. In Proceedings of the 2005 IEEE International Conference on Robotics and Automation, Barcelona, Spain, 18-22 April 2005; pp. 2283-2289.

17. Dollar, A.M.; Herr, H. Design of a quasi-passive knee exoskeleton to assist running. In Proceedings of the 2008 IEEE/RSJ International Conference on Intelligent Robots and Systems, Nice, France, 22-26 September 2008; pp. 747-754.

18. Pratt, J.E.; Krupp, B.T.; Morse, C.J.; Collins, S.H. The RoboKnee: An exoskeleton for enhancing strength and endurance during walking. In Proceedings of the IEEE International Conference on Robotics and Automation, New Orleans, LA, USA, 26 April-1 May 2004; Volume 3, pp. 2430-2435.

19. Karavas, N.C.; Tsagarakis, N.G.; Caldwell, D.G. Design, modeling and control of a series elastic actuator for an assistive knee exoskeleton. In Proceedings of the 2012 4th IEEE RAS \& EMBS International Conference on Biomedical Robotics and Biomechatronics (BioRob), Rome, Italy, 24-27 June 2012; pp. 1813-1819.

20. Noh, J.; Kwon, J.; Yang, W.; Oh, Y.; Bae, J.H. A 4-bar mechanism based for knee assist robotic exoskeleton using singular configuration. In Proceedings of the IECON 2016-42nd Annual Conference of the IEEE Industrial Electronics Society, Florence, Italy, 23-26 October 2016; pp. 674-680.

21. Size Korea. Available online: https://sizekorea.kr/measurement-data/body (accessed on 18 July 2020). 
22. Sibella, F.; Galli, M.; Romei, M.; Montesano, A.; Crivellini, M. Biomechanical analysis of sit-to-stand movement in normal and obese subjects. Clin. Biomech. 2003, 18, 745-750. [CrossRef]

23. Yoshioka, A.; Nagano, R.; Himeno, R.; Fukashiro, S. Computation of the kinematics and the minimum peak joint moments of sit-to-stand movements. Biomed. Eng. 2007, 6, 26. [CrossRef] [PubMed]

24. Delp, S.L.; Anderson, F.C.; Arnold, A.S.; Loan, P.; Habib, A.; John, C.T.; Guendelman, E.; Thelen, D.G. OpenSim: Open-source software to create and analyze dynamic simulations of movement. IEEE Trans. Biomed. Eng. 2007, 54, 1940-1950. [CrossRef] [PubMed]

25. Liu, M.Q.; Anderson, F.C.; Schwartz, M.H.; Delp, S.L. Muscle contributions to support and progression over a range of walking speeds. J. Biomech. 2015, 41, 3243-3252. [CrossRef] [PubMed]

26. Simtk. Available online: https://simtk.org/projects/mspeedwalksims (accessed on 18 July 2020).

27. Winter, D.A. Biomechanics and Motor Control of Human Movement, 4th ed.; University of Waterloo: Waterloo, ON, Canada, 2009; pp. 83-95.

28. Meldrum, D.; Cahalane, E.; Conroy, R.; Fitzgerald, D.; Hardiman, O. Maximum voluntary isometric contraction: Reference values and clinical application. Amyotroph. Lateral Scler. 2007, 8, 47-55. [CrossRef] [PubMed]

29. Zanotto, D.; Akiyama, Y.; Stegall, P.; Agrawal, S.K. Knee Joint Misalignment in Exoskeletons for the Lower Extremities: Effects on User's Gait. IEEE Trans. Robot. 2015, 31, 978-987. [CrossRef]

(C) 2020 by the authors. Licensee MDPI, Basel, Switzerland. This article is an open access article distributed under the terms and conditions of the Creative Commons Attribution (CC BY) license (http://creativecommons.org/licenses/by/4.0/). 